\title{
Mutations in the BCR-ABL1 kinase domain in patients with chronic myeloid leukaemia treated with TKIs or at diagnosis
}

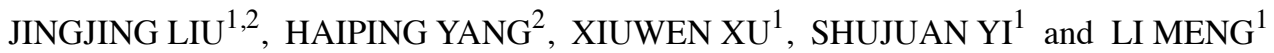 \\ ${ }^{1}$ Department of Hematology, Tongji Hospital of Huazhong University of Science and Technology, \\ Wuhan, Hubei 430030; ${ }^{2}$ Department of Hematology, First Affiliated Hospital of Henan University of \\ Science and Technology, Luoyang, Henan 471000, P.R. China
}

Received March 29, 2019; Accepted January 10, 2020

DOI: $10.3892 / \mathrm{ol} .2020 .11650$

\begin{abstract}
The aim of the present study was to analyse the incidence of mutations in the BCR-ABL1 kinase region in patients with newly diagnosed or treated chronic myeloid leukaemia (CML), and the association between mutations clinicopathological characteristics. Samples were collected for mutation analysis from patients who exhibited tyrosine kinase inhibitor resistance following treatment or were in the accelerated or blast phase at diagnosis. The mutations in the breakpoint cluster region (BCR)-ABL proto-oncogene 1 (ABL1) kinase domain were evaluated using conventional sequencing or ultra-deep sequencing (UDS) of peripheral blood samples. Sanger sequencing and UDS of the cDNA region corresponding to the BCR-ABL1 kinase domain was performed. $\chi^{2}$ test was used to assess the association of categorical variables between the mutated and non-mutated groups. In addition, the Kaplan-Meier method was applied to generate the survival curves. Sequencing detected 28 different mutations in 54 of the $175(30.86 \%)$ patients with CML. A total of $14(8.0 \%)$ patients presented with the T315I mutation, accounting for the largest proportion in the mutated group. Eight patients $(4.6 \%)$ presented with more than one mutation, three $(37.5 \%)$ of whom harboured $\mathrm{T} 315 \mathrm{I}$ coexisting with other mutations, and for nine (5.1\%) patients, the results differed between conventional sequencing and UDS, with the mutations being missed by conventional sequencing. The results form this study suggested that programing mutation analysis in patients with chronic myeloid leukaemia timely may guide the choice of TKIs.
\end{abstract}

Correspondence to: Dr Li Meng, Department of Hematology, Tongji Hospital of Huazhong University of Science and Technology, 1905 Jiefang Avenue, Wuhan, Hubei 430030, P.R. China

E-mail: 3259294525@qq.com

Key words: chronic myeloid leukaemia, mutation, tyrosine kinase inhibitor resistance

\section{Introduction}

Chronic myeloid leukaemia (CML), a malignant disease derived from a haematopoietic stem cell clone, is characterised by the production of the breakpoint cluster region (BCR)-ABL proto oncogene 1 (ABL1) fusion gene and Philadelphia $(\mathrm{Ph})$ chromosome, which are present in $90 \%$ of patients and are well-confirmed diagnostic markers for CML $(1,2)$. The BCR-ABL1 fusion gene encodes an oncoprotein that has an activated tyrosine kinase domain in the ABL region, promotes cell proliferation, causes loss of stromal adhesion and arrests apoptosis by activating downstream pathways, including Janus kinase-signal transducer and activator of transcription (Jak-Stat) and Myc (2,3). Niches in the bone marrow also serve a crucial role in the generation and development of CML (4). Currently, tyrosine kinase inhibitors (TKIs) are applied to treat CML and strongly improve the overall survival (OS) of patients $(3,5)$. The majority of patients achieve a life expectancy close to that of the general population (4). However, there are still some problems during treatment: i) The disease still progresses to an advanced phase (AP) or blast crisis (BC) following treatment with TKIs; ii) a number of patients are resistant to TKIs; and iii) patients cannot endure the side effects of TKIs (6). The present study focused on the mechanisms of disease progression during treatment. Often, resistance can lead to disease progression. Resistance to TKI therapy occurs via BCR-ABL1-dependent and BCR-ABL1-independent mechanisms $(7,8)$. One of the most important factors in the BCR-ABL1-dependent mechanism is the mutation of the BCR-ABL1 kinase domain (KD) (7-9).

TKI resistance is defined based on the European Leukemia Net (ELN) recommendations. Resistance can be divided into primary and secondary categories (2). Primary resistance is characterised by any of the following: No complete haematological response or $\mathrm{Ph}+>95 \%$ by 3 months, BCR-ABL1 $>10 \%$ or $\mathrm{Ph}+>35 \%$ by 6 months, or BCR-ABL1 $>1 \%, \mathrm{Ph}+\geq 1 \%$ or complete cytogenetic response by 18 months (10). Secondary resistance is defined by the loss of a previously documented haematological, cytogenetic or molecular response (1).

TKI resistance has been reported to range between 25-30\% among patients initially treated with imatinib (6). Most mutations in the KD lead to the development of resistance to TKI therapy. 
Most patients switched to potentially more effective TKIs based on their mutation profile and achieved major molecular remission, with the exception of those with the T315I mutation. Reliable investigations are required to screen for mutations in patients who fail TKI therapy.

\section{Patients and methods}

Patients. The present study was a retrospective analysis of 175 patients with CML treated at the Department of Hematology, Tongji Hospital of Huazhong University of Science and Technology. A total of 691 patients with CML visited this institution between January 2009 and December 2016; however, only 177 patients underwent BCR-ABL1 mutation screening. Two patients lacking clinical information were excluded from the analysis. Thus, in the present study, 175 patients were evaluated. The data were collected from patients who underwent mutation screening during TKI treatment or at diagnosis. Mutation analysis was performed as requested when the patients exhibited resistance or were diagnosed at AP or BC. There were nine patients diagnosed at $\mathrm{BC}$ and four at AP. The definitions of the disease phases followed the ELN recommendations (2). Patients were grouped by BCR-ABL1 mutation status. The association between patient clinical information and mutation status was analysed. Patient consent was not required due to the retrospective nature of the study.

BCR-ABL1 mutation analysis. The reduction and mutation of BCR-ABL1 fusion transcripts over time was assessed by conventional sequencing and ultra-deep sequencing (UDS) of peripheral blood samples. UDS refers to deep sequencing that takes advantage of a high-throughput method and focuses on a limited genomic region; UDS can detect mutations that cannot be identified by conventional sequencing (11). The basic principles of UDS and conventional sequencing are similar to those of digital PCR (1). PCR and sequencing were used to detect mutations in the ABL1 kinase region of BCR-ABL1. Total RNA was reverse-transcribed into cDNA and then amplified with Platinum Taq Polymerase High Fidelity (Thermo Fisher Scientific, Inc.). The final reaction volume (20 $\mu \mathrm{l}$ ) included $10 \mu \mathrm{l}$ Mix (Takara Bio, Inc.), $2 \mu \mathrm{l}$ primers, $6.5 \mu \mathrm{l}$ RNase-free $\mathrm{H}_{2} \mathrm{O}$ and $1.5 \mu \mathrm{l} \mathrm{cDNA}$ and was performed as follows: Pre-denaturation at $95^{\circ} \mathrm{C}$ for $10 \mathrm{~min}$, denaturation at $95^{\circ} \mathrm{C}$ for $15 \mathrm{sec}$, annealing at $59^{\circ} \mathrm{C}$ for $30 \mathrm{sec}$, extension at $72^{\circ} \mathrm{C}$ for $30 \mathrm{sec}$, denaturation-annealing-extension step performed 35 cycles. The sequences of the primers (Beijing Tianyi Huiyuan Bioscience \& Technology Inc.) were as follows: Homo BCR-ABL, forward 5'-GATGCTGACCAACTCGTG TG-3', reverse 5'-GTTGGGGTCATTTTCACTGG-3'; and GAPDH, forward 5'-CCACCATGGCAAATTCCATGGCA-3' and reverse 5'-TCTAGACGGCAGGTCAGGTCCACC-3'. The forward primers annealed to BCR exon b2, and the reverse primers annealed to ABL exon 10 (12). After amplification of each single fragment read and mapping to the reference sequence, the results were screened for mutations.

T315I is located in the ATP-binding domain (13), which is the most frequent mutation, and is resistant to currently available TKIs. The frequency of the T315I mutation was analysed in the present study, as well as focusing on compound mutants and polyclonal mutants. Compound mutations are mutations affecting two or more amino acid residues in the same BCR-ABL molecule BCR-ABL molecule (6). Multi-TKI failure leads to the emergence of compound mutants. To date, 60 different compound mutants in BCR-ABL1 have been reported to be associated with TKI resistance $(14,15)$. Polyclonal mutations are defined as two or more missense mutations in different BCR-ABL1 molecules (8).

Statistical analysis. To assess the associations between two groups of categorical variables, $\chi^{2}$ test was used, and age was adjusted by a correction of continuity (in case of $n>40$ and $1 \leq \mathrm{T}<5$ ). OS was calculated from the identification of the mutation until the last follow-up, death or the censor date. To generate survival curves, the Kaplan-Meier method was applied. All calculations were performed using SPSS 17.0 (SPSS, Inc.). $\mathrm{P}<0.05$ was considered to indicate a statistically significant difference.

\section{Results}

Patient characteristics. Table I summarises the clinical characteristics at diagnosis and at mutation screening of the 175 patients analysed in the present study. The characteristics of patients are presented in Table SI. Patients were divided into groups based on BCR-ABL1 mutation status. No differences were observed in the clinical characteristics between the two groups, with the exception of sex; more female patients were present in the BCR-ABL1 mutation group compared with male patients.

Mutation analysis. Patients underwent UDS and conventional sequencing. Different results were detected by USD and conventional sequencing: V253H/M351I/F359V/F359I vs. V253H/M351I; E255K vs. G250E/E255K; F359V vs. none; F359V vs. none; T315I vs. none; T315I vs. none; T315I vs. none; and K365E vs. none. Samples for mutation analysis were collected from patients who presented with TKI resistance, were on first-line TKIs (164 patients, 93.7\%), or were on second-line TKIs (11 patients, 6.3\%). The T315I mutation was identified to account for the largest proportion. A total of 28 patients underwent mutation screening at diagnosis, and mutations were identified in 8 of these 28 patients (4.6\%), which indicated that the mutation exists before treatment with TKIs.

A total of 175 patients underwent BCR-ABL1 mutation screening, and only 54 (30.9\%) patients harboured mutations in the KD, which included 28 different mutations (Fig. 1). Mutations that occurred in the P-loop region included G250E, M244V, L248V, E255K and Y253H, accounting for 36.1\% of the mutations identified in the present study (Table II). The proportion of mutations in the A-loop, contact binding site and SH2 domain is presented in Table II. The number of patients with different types of kinase domain mutations is presented in Table III. Of note, eight patients harboured more than one mutation: V253H/M351I, G250E/E255K, M351T/F359V/H396R, E255K/V299L/T315I, T315I/Y253H, F311L/F317L, L248V/Y253H/T315I and V260A/H295Y. However, it could not be confirmed whether these mutations were compound or polyclonal in this retrospective study (16). Of the eight patients with BCR-ABL1 compound or polyclonal 
Table I. Characteristics of 175 patients with chronic myeloid leukaemia at the time of mutation screening.

\begin{tabular}{|c|c|c|c|c|}
\hline \multirow[b]{2}{*}{ Variable } & \multirow[b]{2}{*}{$\mathrm{N}$} & \multicolumn{2}{|c|}{ BCR-ABL1 mutation } & \multirow[b]{2}{*}{ P-value } \\
\hline & & Present $(n=54)$ & Absent $(n=121)$ & \\
\hline Sex & & & & $<0.001^{\mathrm{a}}$ \\
\hline Male & 111 & 22 & 89 & \\
\hline Female & 64 & 32 & 32 & \\
\hline Age, years & & & & 0.320 \\
\hline Mean, 43 (range, 3-76) & 169 & $44(3-71)$ & $42(3-76)$ & \\
\hline Missing & 6 & & & \\
\hline Disease phase at diagnosis & & & & 0.253 \\
\hline $\mathrm{CP}$ & 162 & 48 & 114 & \\
\hline AP & 4 & 1 & 3 & \\
\hline $\mathrm{BC}$ & 9 & 5 & 4 & \\
\hline Time between TKI and mutation screen, month & & & & 0.140 \\
\hline Mean, 21.5 (range, 0-99) & 166 & $17.3(0-92)$ & $23.5(0-99)$ & \\
\hline Missing, $\mathrm{n}$ & 9 & 4 & 5 & \\
\hline Type of transcription & & & & 0.230 \\
\hline P210 & 167 & 50 & 117 & \\
\hline $\mathrm{P} 230$ & 8 & 4 & 4 & \\
\hline Choice of TKI generation at diagnosis & & & & 0.790 \\
\hline First & 164 & 51 & 113 & \\
\hline Second & 11 & 3 & 8 & \\
\hline Additional chromosome & & & & 0.989 \\
\hline Present & 39 & 12 & 27 & \\
\hline Absent & 136 & 42 & 94 & \\
\hline
\end{tabular}

${ }^{\mathrm{a}} \mathrm{P}<0.05$. CP, chronic phase; AP, accelerated phase; BC, blast crisis; m, month; missing, loss of follow-up; P210, M-type b3a2 (e14a2), b2a2 (e13a2); P230, $\mu$-type e19a2; TKI, tyrosine kinase inhibitor; additional chromosome, in addition to the Philadelphia chromosome attached to other abnormal chromosomes.

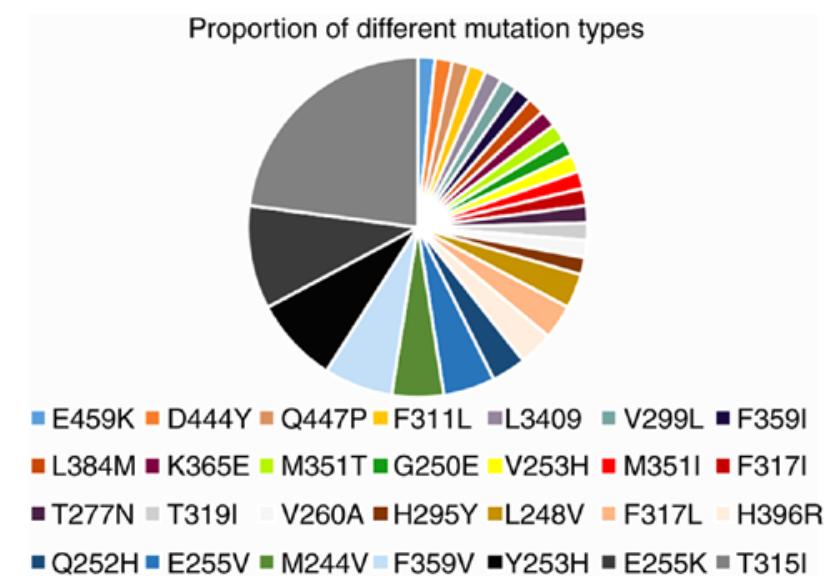

Figure 1. Proportion of different types of mutations in the present study.

mutations, including the T315I mutation, three exhibited variable sensitivity to clinically available TKIs (data not shown), suggesting that one or more TKIs may represent a rational treatment option. These patients harboured detectable mutations and elevated BCR-ABL1 transcription following multi-TKI treatment. The patients with the E255K/V299L/T315I and L248V/Y253H/T315I mutations died. At the end of the follow-up period, the majority of the patients were in $\mathrm{BC}$, treated with combination chemotherapy and TKI with a poor response to treatment.

Survival analysis. The median follow-up time for all patients in the present study, from the day of mutation detection to the last follow-up date, was 22 months (range, 2-88 months). The OS rates of patients in the BCR-ABL1 mutation-positive and negative groups were 80 and $100 \%$, respectively $(\mathrm{P}<0.01)$. The OS rates of the groups with or without the T315I mutation were 47 and $98 \%$, respectively $(\mathrm{P}<0.01$; Fig. $2 \mathrm{~A}$ and $\mathrm{B})$. These results indicated that the OS rate of patients with the T315I mutation was notably decreased.

T315I mutation. The T315 residue is the gatekeeper, and when it is mutated to T315I, the entrance of a TKI into the hydrophobic pocket is blocked, while still allowing access to ATP (17). The T315I mutation, which is the most challenging mutation, indicates a poor prognosis (18). Unfortunately, patients with the T315I mutation are usually resistant to all available TKIs (imatinib, bosutinib, nilotinib and 
Table II. The proportion of mutations that occur in distinct regions.

\begin{tabular}{llc}
\hline Region & \multicolumn{1}{c}{ Mutation } & Proportion, \% \\
\hline P-loop & G250E, M244V, & 36.1 \\
& L248V, E255K/V, & \\
& Y253H & \\
Contact binding site & T315I & 23 \\
& F311L & 1.6 \\
& F317L/I & 3.3 \\
SH2 domain & M351T/I & 3.3 \\
& F359V & 8.2 \\
A-loop & H396R & 3.3 \\
Other ${ }^{\text {a }}$ & H295Y, K356E, & 21.2 \\
& V260A, Q447P, & \\
& T319I, V253H, & \\
& L384M, D444Y, & \\
& E459K, V299L, \\
& T277N, L3409 \\
\hline
\end{tabular}

${ }^{\mathrm{a}}$ Low frequency mutations.

Table III. Number of patients with different types of kinase domain mutations.

\begin{tabular}{lc} 
Kinase domain mutation & Number of patients $(\mathrm{n}=61)$ \\
\hline T315I & 14 \\
E255K & 6 \\
Y253H & 5 \\
F359V & 4 \\
E255V & 3 \\
M244V & 3 \\
Other $^{\text {a }}$ & 26 \\
\hline
\end{tabular}

${ }^{\mathrm{a}}$ Low frequency mutations.

dasatinib) but may benefit from ponatinib (18-20) or allogeneic haematopoietic stem cell transplantation (allo-HSCT). Of the 14 patients who presented with the T315I mutation, one patient achieved complete molecular remission after allo-HSCT (19). The results demonstrated that patients with the T315I mutation could be treated with allo-HSCT if a suitable donor was available. A total of six patients were treated with a combination of a TKI and chemotherapy and died in 1-2.5 years $(18,21)$. Third-generation TKIs such as ponatinib may have efficacy against the T315I mutation (21). However, it is difficult to obtain ponatinib in China. In the present study, two patients were treated with ponatinib and exhibited a good response; however, these results were temporary. Additionally, these two patients presented with cardiovascular toxicity, which may have been an adverse effect of ponatinib.
Karyotype analysis. Sufficient sample material was available to examine the Karyotype. The Philadelphia chromosome (Ph) is a reciprocal translocation between the Abelson leukemia virus (ABL) oncogene from long arm of chromosome 9 and the breakpoint cluster region (BCR) from long arm of chromosome 22. Additional chromosome refers to in addition to the Philadelphia chromosome attached to other abnormal chromosomes.

In the present study, 39 patients presented with additional chromosomes (in addition to the Philadelphia chromosome attached to other abnormal chromosomes), and six of these patients had the T315I mutation. No association was identified between the T315I mutation and additional chromosome in patients with CML $(\mathrm{P}=0.086)$.

\section{Discussion}

This study analyzed data from 175 patients with CML who underwent mutation screening. A total of 162 (92.6\%) patients were diagnosed at the chronic phase (CP), 48 of whom harboured detectable mutations, and four were diagnosed at AP, among which one had a mutation. A total of nine patients, who were diagnosed at BC, were tested for mutations. However, the mutation was not associated with disease phase.

The T315I mutation was detected in patients. Among all patients, T315I was the most frequently detected mutation in the present study, and this mutation was also associated with OS. The patients who presented with the T315I mutation responded poorly to chemotherapy. Only one patient, aged 28 years at diagnosis, achieved molecular remission following allo-HSCT. In addition, two patients benefited from ponatinib, although with notable toxicity. A total of five patients died despite receiving chemotherapy. Ponatinib has been described as an effective treatment for patients with the T315I mutation (22). These results were similar to those reported in the literature (6).

In the present study, the results of conventional sequencing were different from those of UDS in eight patients. This result confirmed that UDS exhibits higher sensitivity for mutations compared with conventional sequencing. The K365E mutation, which was detected by UDS, was not associated with sensitivity to any type of TKI according to available literature. The patient with the K365E mutation was treated with dasatinib and achieved a complete cytogenetic response.

In the present study, eight patients presented with more than one mutation. Of note, three patients had T315I mutations in combination with other mutations, and two of these patients died; however, it is unknown whether these were polyclonal or compound mutations. The results from the present study demonstrated that the prognosis of patients with multiple BCR-ABL1 mutations is poor, especially for those with T315I compound or polyclonal mutations. In the present study, the overall survival of patients with compound mutations was not analysed due to the limited number of cases.

The emergence of TKI resistance can be divided into BCR-ABL-dependent and BCR-ABL-independent processes. BCR-ABL-dependent processes includeoverexpression of the BCR-ABL kinase and mutations in BCR-ABL domain. BCR-ABL-independent processes include activation of alternative signalling pathways (such as JAK/STAT) and overexpression of efflux transporters or downregulation 
A
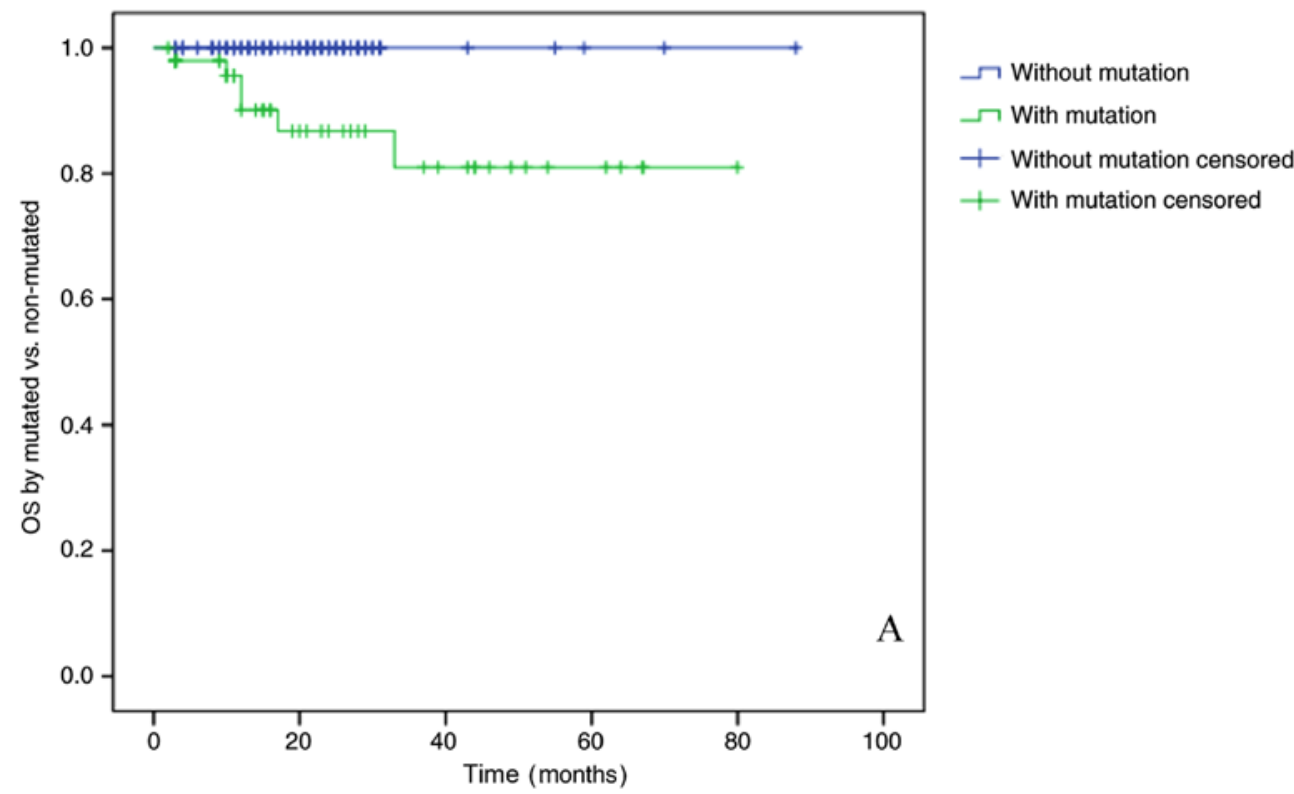

B

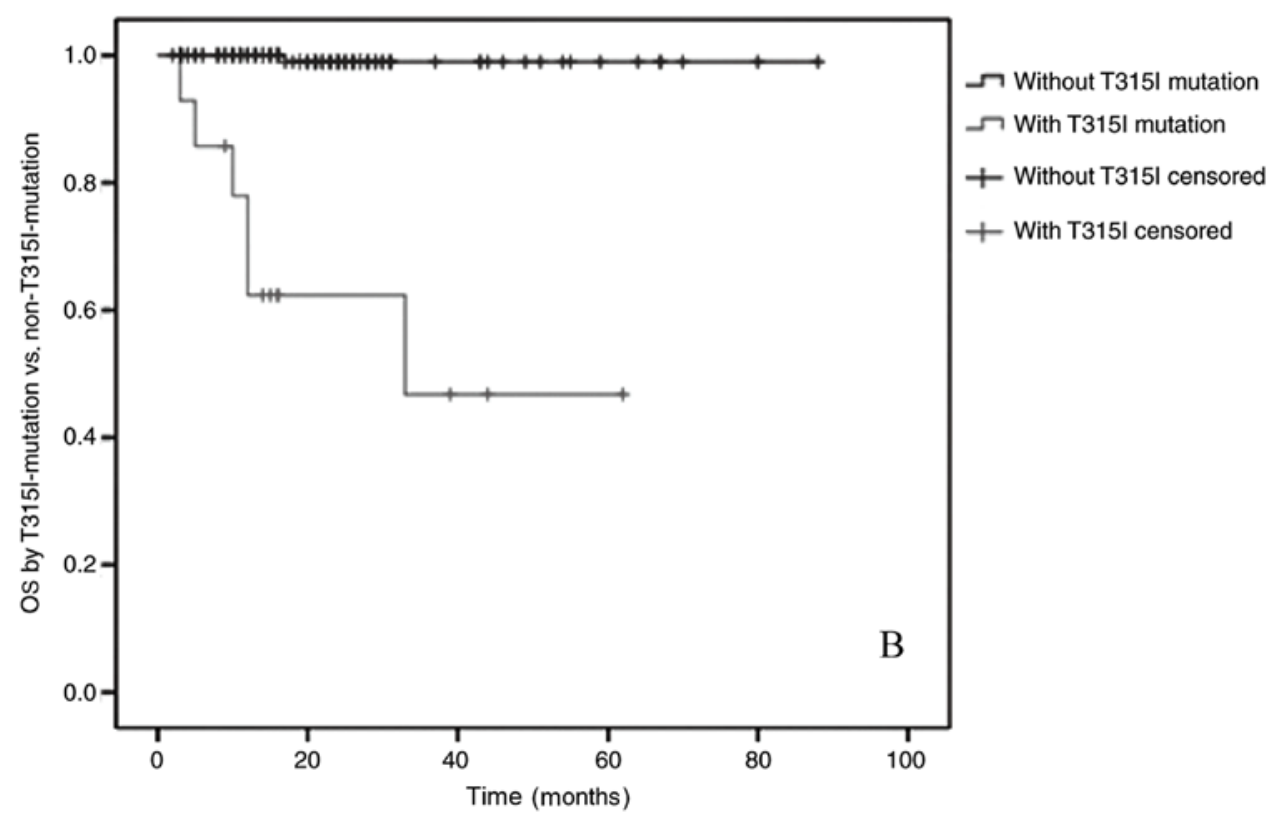

Figure 2. (A) OS of 175 patients with CML based on mutation status (mutated vs. non-mutated) and (B) T315I mutation status (with T315I mutation vs. without T315I mutation). $\mathrm{P}<0.01$. CML, chronic myeloid leukaemia; OS, overall survival.

of influx transporters leading to low TKI levels in the cell. Mutations in the BCR/ABL KD are the most important of these mechanisms. Mutations in the KD leading to resistance to TKI therapy have a much higher incidence in patients in $\mathrm{BC}$ compared with patients in $\mathrm{CP}(23)$. Patients with mutations in $\mathrm{CP}$ exhibit a higher incidence of progression to $\mathrm{AP}$ or $\mathrm{BC}$. Thus, mutations can provide information for patients who do not achieve optimal responses to TKIs $(10,11)$. Currently, a number of options of therapeutic TKI are available, depending on the mutations associated with TKI resistance $(24,25)$. Acquired mutations prevent TKIs from binding or lead to a decrease in TKI sensitivity (7). Subsets of these mutations, which have been reported in certain TKI-resistant cases, remain sensitive to one or more first-generation (imatinib) or second-generation (nilotinib, dasatinib, bosutinib and ponatinib) TKIs (2). Distinct mutations exhibit different levels of sensitivity to TKIs. In the present study, TKIs were selected based on the nature of the mutation; for example, dasatinib has activity against $\mathrm{T} 315 \mathrm{I}$ and $\mathrm{F} 317 \mathrm{~L} / \mathrm{C}$, whereas $\mathrm{T} 315 \mathrm{I}$ and F359V/C have limited sensitivity to nilotinib (19,26-28). However, the unique mutation T315I affects the topology of the ATP binding region and is not responsive to any available TKI, except ponatinib (17). Thus, the detection of mutations can aid with selecting the TKI and influence the prognosis of patients with CML, who can be switched to different TKIs based on the detected mutation.

CML can be regarded as a chronic illness due to the contribution of TKIs; however, a small number of patients still experience disease progression. Thus, more clinical case analyses are required to better understand CML. 


\section{Acknowledgements}

Not applicable.

\section{Funding}

No funding was received.

\section{Availability of data and materials}

The datasets used and/or analysed during the current study are available from the corresponding author on reasonable request.

\section{Authors' contributions}

JL collected and analyzed data about patients and methods, and completed the draft. HY, XX and SY collected data about the patients and helped analyzing data. LM made substantial contributions to conception and design of the study and revised and wrote the final version of the manuscript. All authors read and approved the final manuscript.

\section{Ethics approval and consent to participate}

Not applicable.

\section{Patient consent for publication}

Not applicable.

\section{Competing interests}

The authors declare that they have no competing interests.

\section{References}

1. Machova Polakova K, Kulvait V, Benesova A, Linhartova J, Klamova H, Jaruskova M, de Benedittis C, Haferlach T, Baccarani M, Martinelli G, et al: Next-generation deep sequencing improves detection of BCR-ABL1 kinase domain mutations emerging under tyrosine kinase inhibitor treatment of chronic myeloid leukemia patients in chronic phase. J Cancer Res Clin Oncol 141: 887-899, 2015.

2. Kaleem B, Shahab S, Ahmed N and Shamsi TS: Chronic myeloid leukemia-prognostic value of mutations. Asian Pac J Cancer Prev 17: 7415-7423, 2015

3. Apperley JF: Chronic myeloid leukaemia. Lancet 385: 1447-1459, 2015.

4. Loscocco F, Visani G, Galimberti S, Curti A and Isidori A: BCR-ABL independent mechanisms of resistance in chronic myeloid leukemia. Front Oncol 9: 939, 2019.

5. Pasic I and Lipton JH: Current approach to the treatment of chronic myeloid leukaemia. Leuk Res 55: 65-67, 2017.

6. Eide CA and O'Hare T: Chronic myeloid leukemia: Advance in understanding disease biology and mechanisms of resistance to tyrosine kinase inhibitors. Curr Hematol Malig Rep 10: 158-166, 2015.

7. Soverini S, de Benedittis C, Mancini M and Martinelli G: Mutations in the BCR-ABL1 kinase domain and elsewhere in chronic myeloid leukemia. Clin Lymphoma Myeloma Leuk S1 (Suppl): S120-S128, 2015.

8. Yang K and Fu LW: Mechanisms of resistance to BCR-ABL TKIs and the therapeutic strategies: A review. Crit Rev Oncol Hematol 93: 277-292, 2015.
9. Balabanov S, Braig M and Brümmendorf TH: Current aspects in resistance against tyrosine kinase inhibitors in chronic myelogenous leukemia. Drug Discov Today Technol 11: 89-99, 2014.

10. Baccarani M, Castagnetti F, Gugliotta G and Rosti G: A review of the European LeukemiaNet recommendations for the management of CML. Ann Hematol 94 (Suppl 2): S141-S147, 2015.

11. Soverini S, De Benedittis C, Mancini M and Martinelli G: Present and future of molecular monitoring in chronic myeloid leukaemia. Br J Haematol 173: 337-349, 2016

12. Pagnano KB, Bendit I, Boquimpani C, De Souza CA, Miranda EC, Zalcberg I, Larripa I, Nardinelli L, Silveira RA, Fogliatto L, et al: BCR-ABL mutations in chronic myeloid leukemia treated with tyrosine kinase inhibitors and impact on survival. Cancer Invest 33: 451-458, 2015.

13. Cagnetta A, Garuti A, Marani C, Cea M, Miglino M, Rocco I, Palermo C, Fugazza G, Cirmena G, Colombo N, et al: Evaluating treatment response of chronic myeloid leukemia: Emerging science and technology. Curr Cancer Drug Targets 13: 779-790, 2013.

14. Shah NP, Skaggs BJ, Branford S, Hughes TP, Nicoll JM, Paquette RL and Sawyers CL: Sequential ABL kinase inhibitor therapy selects for compound drug-resistant BCR-ABL mutations with altered oncogenic potency. J Clin Invest 117: 2562-2569, 2007.

15. Khorashad JS, Kelley TW, Szankasi P, Mason CC, Soverini S, Adrian LT, Eide CA, Zabriskie MS, Lange T, Estrada JC, et al: BCR-ABL1 compound mutations in tyrosine kinase inhibitor-resistant CML: Frequency and clonal relationships. Blood 121: 489-498, 2013

16. Gibbons DL, Pricl S, Posocco P, Laurini E, Fermeglia M, Sun H, Talpaz M, Donato N and Quintás-Cardama A: Molecular dynamics reveal BCR-ABL1 polymutants as a unique mechanism of resistance to PAN-BCR-ABL1 kinase inhibitor therapy. Proc Natl Acad Sci USA 111: 3550-3555, 2014.

17. Miller GD, Bruno BJ and Lim CS: Resistant mutations in CML and Ph+ALL-role of ponatinib. Biologics 8: 243-254, 2014.

18. Poch Martell M, Sibai H, Deotare U and Lipton JH: Ponatinib in the therapy of chronic myeloid leukemia. Expert Rev Hematol 9: 923-932, 2016.

19. Kujak C and Kolesar JM: Treatment of chronic myelogenous leukemia. Am J Health Syst Pharm 73: 113-120, 2016.

20. Modugno M: New resistance mechanisms for small molecule kinase inhibitors of Abl kinase. Drug Discov Today Technol 11: 5-10, 2014.

21. Haznedaroglu IC: Drug therapy in the progressed CML patient with multi-TKI failure. Mediterr J Hematol Infect Dis 7: e2015014, 2015.

22. Patel AB, O'Hare T and Deininger MW: Mechanisms of resistance to ABL kinase inhibition in chronic myeloid leukemia and the development of next generation ABL kinase inhibitors. Hematol Oncol Clin North Am 31: 589-612, 2017.

23. Apperley JF: Part I: Mechanisms of resistance to imatinib in chronic myeloid leukemia. Lancet Oncol 8: 1018-1029, 2007.

24. Corbin AS, La Rosée P, Stoffregen EP, Druker BJ and Deininger MW: Several BCR-ABL kinase domain mutants associated with imatinib mesylate resistance remain sensitive to imatinib. Blood 101: 4611-4614, 2003.

25. Hochhaus A, Schenk T, Erben P, Ernst T, La Rosée P and Müller MC: Cause and management of therapy resistance. Best Pract Res Clin Haematol 22: 367-379, 2009.

26. Hehlmann R, Saußele S, Voskanyan A and Silver RT: Management of CML-blast crisis. Best Pract Res Clin Haematol 29: 295-307, 2016.

27. Soverini S, De Benedittis C, Mancini M and Martinelli G: Best practices in chronic myeloid leukemia monitoring and management. Oncologist 21: 626-633, 2016.

28. Morozova EV, Vlasova YY, Pryanishnikova MV, Lepik KV and Afanasyev BV: Efficacy of dasatinib in a CML patient in blast crisis with F317L mutation: A case report and literature review. Biomarker Insights 10 (Suppl 3): S43-S47, 2015.

This work is licensed under a Creative Commons Attribution-NonCommercial-NoDerivatives 4.0 International (CC BY-NC-ND 4.0) License. 